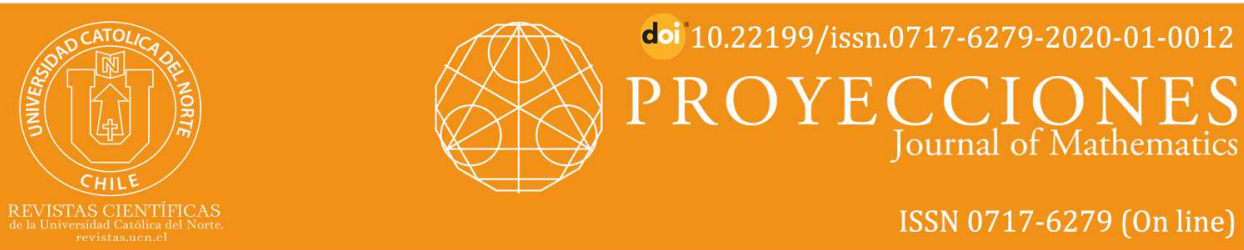

\title{
Lie symmetry analysis and traveling wave solu- tions of equal width wave equation
}

\author{
Antim Chauhan ${ }^{1}$ (D) orcid.org/0000-0002-5430-1437 \\ Rajan Arora ${ }^{2}$ (i) orcid.org/0000-0003-1109-122X \\ Amit Tomar ${ }^{3}$ (D) orcid.org/0000-0002-6293-2888 \\ Indian Institute of Technology Roorkee, Dept of Appl. Sci. and Engineer., Roorkee, UT, India. \\ 1曰antimchauhan1@gmail.com \\ 2四rajanfpt@iitr.ernet.in \\ ${ }^{3}$ Amity University, Dept. of Mathematics, Noida, UP, India. \\ 口amitmath14@gmail.com \\ Received: January2019 | Accepted: October 2019
}

\section{Abstract:}

We obtained the power series solution and the traveling wave solutions of equal width wave equation by using the Lie symmetry method. The fundamental idea behind the symmetry transformation method is that it reduces one independent variables in a system of PDEs by utilizing Lie symmetries and surface invariance condition. We first obtained the infinitesimals and commutation table with the help of MAPLE software. Lie symmetry transformation method (STM) has been applied on EWW equation and converted it into various nonlinear ODEs. Then, the tanh method and the power series method have been applied for solving the reduced nonlinear ordinary differential equations (ODEs). Convergence of the power series solutions has also been shown.

Keywords: Equal width wave (EWW) equation; Lie symmetry analysis method; Power series solutions; Tanh method; Symbolic computation.

MSC (2010): 74J99; 76M60.

\section{Cite this article as (IEEE citation style)}

A. Chauhan, R. Arora , and A. Tomar,"Lie symmetry analy-

sis and traveling wave solutions of equal width wave equa-

tion", Proyecciones (Antofagasta, On line), vol. 39, no. 1, pp.

179-198, Feb. 2020, doi: 10.22199/issn.0717-6279-

2020-01-0012. [Accessed dd-mm-yyyy].

Article copyright: (C) 2020 Antim Chauhan, Rajan Arora and Amit Tomar. This is an open access article distributed under the terms of the Creative Commons Licence, which permits unrestricted use and distribution provided the original author and source are credited.

(cc) BY 


\section{Introduction}

The nonlinear evolution equations (NLEEs) are experienced in many fields of engineering, mathematics, physics, chemistry and biology. Also, the nonlinear evolution equations are extensively used to describe the complex physical phenomena in various fields of science and engineering, mainly in fluid mechanics, solid state physics, plasma wave and plasma physics and chemical physics etc. In this work, we are going to solve a nonlinear evolution equation known as the equal width wave equation [1]. The equal width wave $(\mathrm{EWW})$ equation is

$$
u_{t}+u u_{x}-\mu u_{x x t}=0,
$$

where $\mu$ is a positive parameter.

The solution of nonlinear partial differential equation plays an important role in understanding the behavior of complex system. Equal width wave (EWW) equation, introduced by Morrison et al. [2], is of great interest because it is used as the model partial differential equation for the simulation of one dimensional wave propagation in nonlinear media with dispersive process. Various numerical and analytical techniques have been used for finding the solution of the EWW equation.

Ji-Huan He [3] was the first who introduced the variational iteration method in 1998. Yusufoglu and Bekir [4] obtained the numerical solution of EWW equation by using the variational iteration method and compared the obtained result with the solution obtained by the Adomian Decomposition Method. Jun and Xia [5] solved EWW equation by using the mesh-free reproducing kernel particle Ritz method. Kaplan et al. [1] obtained exact traveling wave solution of EWW equation by using the Auxiliary Equation method. Bihloa and Popovych [6] solved the group classification problem for the class of $(1+1)$-dimensional rth order $(r>2)$ linear evolution equations. They also computed exact solutions for equations from the class under consideration using Lie reduction. The method of Lie group analysis is used by Rosca et. al [7] to investigate the steady MHD double-diffusive convection of a viscous and electrically conducting fluid from a permeable vertical stretching/shrinking sheet. Lie symmetry analysis of the $(2+1)$ dimensional dissipative AKNS equation is done by Ma et. al [8]. The researchers $[9,10,11,12,13,14,15]$ inspired us to solve Eq.(1.1) analytically by using the Lie symmetry Method. In the present work, we solve 
the EWW equation by using the Lie Symmetry Method.

The outlines of this paper are as follows. In Sec.2, the general procedure of Lie Symmetry Analysis Method have been discussed. In Sec.3, the Lie point symmetry of the MEW equation (1.1) and the commutation relations of the generators associated with the symmetry are provided by means of the classical method. Also, optimal system of subalgebras are obtained

in Sec.3 by optimal system method. Sec.4 is devoted to the reduction of nonlinear PDE (1.1) into nonlinear ODE by using similarity variable for optimal system of subalgebras of Eq.(1.1). In Sec.5, exact traveling wave solutions of the MEW Eq. (1.1) have been obtained by using tanh Method. Power series solution of MEW equation and their convergence have been proved in Sec.6. Finally, Sec.7 concludes the paper.

\section{General procedure of Lie Symmetry Analysis Method}

In this section, we describe the general strategy of Lie symmetry analysis $[16,17,18]$ to determine the symmetries of any system of nonlinear partial differential equations (NPDEs). Let us consider the general nonlinear system of $\mathrm{k}$-th order partial differential equations (PDEs) in $m$-independent variables say $X=\left(x^{1}, x^{2}, \ldots, x^{m}\right) \in R^{m}$ and $n$-dependent variables say $U=\left(u^{1}, u^{2}, \ldots, u^{n}\right) \in R^{n}$ in the following form:

$$
\Delta_{\nu}\left(X, U^{(k)}\right)=0, \quad \nu=1,2, \ldots, l,
$$

where $U^{(k)}$ represents all order derivatives of $u$ upto $k$.

We consider one parameter Lie group of infinitesimal transformations acting on both the independent and dependent variables of system (2.1), as follows:

$$
\begin{gathered}
\tilde{x}^{i} \rightarrow x^{i}+\epsilon \xi^{i}(X, U)+O\left(\epsilon^{2}\right), \quad i=1,2, \ldots m, \\
\tilde{u}^{j} \rightarrow u^{j}+\epsilon \eta^{j}(X, U)+O\left(\epsilon^{2}\right), \quad j=1,2, \ldots n,
\end{gathered}
$$

where $\epsilon$ is a very small parameter of the transformation, usually taken as $\epsilon<<1$ and $\xi^{i}$ and $\eta^{j}$ are the infinitesimals of the transformation with respect to the independent and dependent variables, respectively. 
The infinitesimal generator of the above transformation can be written as

$$
V=\sum_{i=1}^{m} \xi^{i}(X, U) \frac{\partial}{\partial x^{i}}+\sum_{j=1}^{n} \eta^{j}(X, U) \frac{\partial}{\partial u^{j}} .
$$

The surface invariance condition of the system (2.1) is given as

$$
\begin{array}{r}
\operatorname{Pr}^{(k)} V\left[\Delta_{\nu}\left(X, U^{(k)}\right)\right]=0, \quad \nu=1,2, \ldots, l, \\
\text { whenever } \quad \Delta_{\nu}\left(X, U^{(k)}\right)=0,
\end{array}
$$

where $\operatorname{Pr}^{(k)}$ represents the $k$-th order prolongation of the infinitesimal generator $V$, which is defined as

$$
\operatorname{Pr}^{(k)} V=V+\sum_{r=1}^{n} \sum_{J} \eta_{r}^{J}\left(X, U^{(k)}\right) \frac{\partial}{\partial u_{J}^{r}},
$$

where $J=\left(j_{1}, j_{2}, \ldots j_{s}\right), 1 \leq j_{s} \leq m, 1 \leq s \leq k$ and the sum varies over all J's of order $0<J \leq k$. If $J=s$, the coefficient $\eta_{r}^{J}$ of $\frac{\partial}{\partial u_{J}^{r}}$ will only depend on the $s$-th or less order derivatives of $U$, and we have

$$
\eta_{r}^{J}\left(X, U^{(k)}\right)=D_{J}\left(\eta_{r}-\sum_{i=1}^{m} \xi^{i} u_{i}^{r}\right)+\sum_{i=1}^{m} \xi^{i} \frac{\partial}{\partial u_{J, i}^{r}},
$$

where $u_{i}^{r}=\frac{\partial u^{r}}{\partial x^{i}}$ and $u_{J, i}^{r}=\frac{\partial u_{J}^{r}}{\partial x^{i}}$.

The infinitesimal symmetries form a Lie algebra under the usual Lie bracket.

\section{Lie Symmetry Analysis of Equal Width Wave Equation}

In this section, we applied the Lie group method on equation (1.1). Let us consider one parameter $(\epsilon<<1)$ Lie group of infinitesimal transformations for the variables $x, t$ and $u$ as follows:

$$
\begin{gathered}
\tilde{x}=x+\epsilon \xi(x, t, u)+O\left(\epsilon^{2}\right), \\
\tilde{t}=t+\epsilon \tau(x, t, u)+O\left(\epsilon^{2}\right), \\
\tilde{u}=u+\epsilon \eta(x, t, u)+O\left(\epsilon^{2}\right),
\end{gathered}
$$


where $\xi, \tau$ and $\eta$ are the infinitesimals. For more details, we give the reference of Olver [16] and Bluman \& Cole [18]. The infinitesimal generator of equation (1.1) is as follows:

$$
V=\xi(x, t, u) \frac{\partial}{\partial x}+\tau(x, t, u) \frac{\partial}{\partial t}+\eta(x, t, u) \frac{\partial}{\partial u} .
$$

The third prolongation is defined as

$$
\begin{aligned}
& \operatorname{Pr}^{(3)} V=\xi \frac{\partial}{\partial x}+\tau \frac{\partial}{\partial t}+\eta \frac{\partial}{\partial u}+\left[\eta^{x}\right] \frac{\partial}{\partial u_{x}}+\left[\eta^{t}\right] \frac{\partial}{\partial u_{t}}+\left[\eta^{x x}\right] \frac{\partial}{\partial u_{x x}}+\left[\eta^{x t}\right] \frac{\partial}{\partial u_{x t}}+ \\
& {\left[\eta^{t t}\right] \frac{\partial}{\partial u_{t t}}+\left[\eta^{x x x}\right] \frac{\partial}{\partial u_{x x x}}+\left[\eta^{x x t}\right] \frac{\partial}{\partial u_{x x t}}+\left[\eta^{x t t}\right] \frac{\partial}{\partial u_{x t t}}+\left[\eta^{t t t}\right] \frac{\partial}{\partial u_{t t t}}}
\end{aligned}
$$

By applying third prolongation $\left(\operatorname{Pr}^{(3)} V\right)$ of vector $V$ to the equation (1.1), we obtain the surface invariance condition, which is given by the following equation:

$$
\eta u_{x}+\left[\eta^{t}\right]+u\left[\eta^{x}\right]-\mu\left[\eta^{x x t}\right]=0
$$

where $\left[\eta^{t}\right],\left[\eta^{x}\right]$ are the first extensions of $V$ for the dependent variable $u$ and $\left[\eta^{x x t}\right]$ is the third extension of $V$ for the dependent variable $u$.

By putting the values of coefficients $\left[\eta^{t}\right],\left[\eta^{x}\right],\left[\eta^{x x t}\right]$ and making use of equation (1.1) into (3.3), we get an equation in terms of partial derivatives of $\xi, \eta$ and $\tau$. Then, we equate the coefficients of various monomials to zero and get the system of PDEs. We find infinitesimals by solving the obtained system of PDEs. These infinitesimals can be determined by doing calculation either manually or by using software MAPLE or MATHEMATICA. Here, we obtained these infinitesimals by using MAPLE 15 as follows:

$$
\xi=c_{3}, \quad \tau=c_{1} t+c_{2}, \quad \eta=-c_{1} u,
$$

where $c_{1}, c_{2}$ and $c_{3}$ are the arbitrary constants.

The Lie algebra of infinitesimal symmetries of equation (1.1) is spanned by the following vector fields:

$$
V_{1}=t \frac{\partial}{\partial t}-u \frac{\partial}{\partial u}, \quad V_{2}=\frac{\partial}{\partial t}, \quad V_{3}=\frac{\partial}{\partial x}
$$


The set $\left\{V_{1}, V_{2}, V_{3}\right\}$ is closed under Lie bracket. The commutation relations of Lie algebra between $V_{1}, V_{2}, V_{3}$ are shown in Table 1.

Table 1

Commutation Table of Lie Algebra.

\begin{tabular}{|l|lll|}
\hline$\left[V_{i}, V_{j}\right]$ & $V_{1}$ & $V_{2}$ & $V_{3}$ \\
\hline$V_{1}$ & 0 & $-V_{2}$ & 0 \\
$V_{2}$ & $V_{2}$ & 0 & 0 \\
$V_{3}$ & 0 & 0 & 0 \\
\hline
\end{tabular}

In the Commutation Table $\mathbf{1}$, the (i, j)-th entry is taken as the value of Lie bracket which is defined as $\left[V_{i}, V_{j}\right]=V_{i} V_{j}-V_{j} V_{i}$. Observing the table, we can state that the given algebra is solvable, where $V_{1}$ is the casimir operator. Obviously, the table is skew-symmetric with zero diagonal elements and all $V_{i}^{\prime} s, \quad i=1,2,3$ are linearly independent.

Now, corresponding to the different values of constants $c_{i} \quad(i=1,2,3)$, we consider the following different seven infinitesimal generators:

Case 1. $c_{1}=1, c_{2}=0, c_{3}=0$, we get infinitesimal generator: $V_{1}=t \frac{\partial}{\partial t}-u \frac{\partial}{\partial u}$.

Case 2. $c_{1}=0, c_{2}=1, c_{3}=0$, we get infinitesimal generator: $V_{2}=\frac{\partial}{\partial t}$.

Case 3. $c_{1}=0, c_{2}=0, c_{3}=1$, we get infinitesimal generator: $V_{3}=\frac{\partial}{\partial x}$.

Case 4. $c_{1}=1, c_{2}=0, c_{3}=1$, we get infinitesimal generator: $V_{4}=\frac{\partial}{\partial x}+t \frac{\partial}{\partial t}-u \frac{\partial}{\partial u}$.

Case 5. $c_{1}=1, c_{2}=1, c_{3}=0$, we get infinitesimal generator: $V_{5}=(t+1) \frac{\partial}{\partial t}-u \frac{\partial}{\partial u}$.

Case 6. $c_{1}=1, c_{2}=1, c_{3}=1$, we get infinitesimal generator: $V_{6}=\frac{\partial}{\partial x}+(t+1) \frac{\partial}{\partial t}-u \frac{\partial}{\partial u}$. 
Case 7. $c_{1}=0, c_{2}=-k, c_{3}=d$, we get infinitesimal generator: $V_{7}=d \frac{\partial}{\partial x}-k \frac{\partial}{\partial t}$.

Next, we search for the optimal system of the one dimensional subalgebras for the Eq. (1.1) based on method in [16, 19].

\section{Optimal System of Subalgebras :}

According to the theory of $[16,19,20]$ an optimal system of one parameter subgroup is the list of conjugacy inequivalent one parameter subgroup with the property that any other subgroup is conjugate to precisely one subgroup in the list. Similarly, a list of one parameter subalgebras forms an optimal system if every one parameter subalgebra is equivalent to a unique member of the list under some element of the adjoint representation. Thus for one dimensional subalgebras, this classification problem is essentially the same as the problem of classifying the orbits of the adjoint representation. In this paper, we are interested only on the symmetry algebra of the equal width wave equation which is spanned by the vector fields $V_{1}, V_{2}$ and $V_{3}$. Every one dimensional subalgebra is determined by a non zero vector of the form:

$$
V=c_{1} V_{1}+c_{2} V_{2}+c_{3} V_{3} .
$$

where $c_{i}$ are the arbitrary constants. We try to simplify many of the coefficients $c_{i}$ as far as possible through judicious application of adjoint maps to $V$. For this purpose we need to find adjoint representation, which can be obtain by using the following Lie series

$\operatorname{Ad}\left(\exp \left(\epsilon V_{i}\right)\right) V_{j}=V_{j}-\epsilon\left[V_{i}, V_{j}\right]+\frac{\epsilon^{2}}{2}\left[V_{i},\left[V_{i}, V_{j}\right]\right]-\cdots$.

Adjoint representation of vector fields of modified equal width wave equation are given in the table below: 
Table 2

Adjoint Representation of Lie Algebra of Eq. (1)

\begin{tabular}{|l|lll|}
\hline$\left[V_{i}, V_{j}\right]$ & $V_{1}$ & $V_{2}$ & $V_{3}$ \\
\hline$V_{1}$ & $V_{1}$ & $(1+\epsilon) V_{2}$ & $V_{3}$ \\
$V_{2}$ & $V_{1}-\epsilon V_{2}$ & $V_{2}$ & $V_{3}$ \\
$V_{3}$ & $V_{1}$ & $V_{2}$ & $V_{3}$ \\
\hline
\end{tabular}

We obtained the following optimal system for equation (1.1), by using the optimal system method:

(1). $V_{1}$

(2). $V_{2}$

(3). $V_{3}$

(4). $V_{3}+V_{1}$

(5). $V_{3}-V_{1}$.

From the above obtained optimal system it is clear that the symmetry $(x ; t ; u) \rightarrow(-x ; t ; u)$; will map (4) to $(5)$; so, we analysis reductions and exact solutions of the remaining four essential vector fields in the optimal system.

\section{Symmetry Reductions and Exact Solution of EWW Equa- tion}

Vector field (1) $V_{1}$

Corresponding to the infinitesimal generator $V_{1}=t \frac{\partial}{\partial t}-u \frac{\partial}{\partial u}$, we have the following characteristic equation: $\frac{d x}{0}=\frac{d t}{t}=\frac{d u}{-u}$, thus by taking the appropriate combinations from this characteristic equation, we get the similarity variable and function as:

$$
\begin{array}{r}
X=x, \\
u=\frac{F(X)}{t},
\end{array}
$$

where $X$ is the similarity variable, $F$ is the similarity function and $u$ is the group invariant solution.

By making use of Eqs.(4.1)-(4.2) in Eq.(1.1), we obtained the following reduced equation: 


$$
-F+F F_{X}+\mu F_{X X}=0 .
$$

The equation (4.3) is a nonlinear ordinary differential equation (ODE). In order to solve this nonlinear ODE, we used the power series method and obtained the exact group invariant solution of Eq.(1.1) in section 6 .

Vector field (2) $V_{2}$

Corresponding to the infinitesimal generator $V_{2}=\frac{\partial}{\partial t}$, we have the following characteristic equation : $\frac{d x}{0}=\frac{d t}{1}=\frac{d u}{0}$, thus by taking appropriate combinations from this characteristic equation, we get the similarity variable and function as:

$$
\begin{array}{r}
X=x, \\
u=F(X),
\end{array}
$$

where $u$ is the group invariant solution of Eq.(1.1). Using Eqs.(4.4)-(4.5), Eq.(1.1) reduces to the following ODE:

$$
F F_{X}=0 \Rightarrow F_{X}=0,
$$

By solving the equation (4.6), we get $F=B_{1}$, which implies $u=B_{1}$, where $B_{1}$ is an arbitrary constant. In this case, we get the trivial solution of Eq.(1.1).

\section{Vector field (3) $V_{3}$}

Corresponding to the infinitesimal generator $V_{3}=\frac{\partial}{\partial x}$, we have the following characteristic equation : $\frac{d x}{1}=\frac{d t}{0}=\frac{d u}{0}$, thus by taking appropriate combinations from this characteristic equation, we get similarity variable and function as:

$$
\begin{array}{r}
T=t, \\
u=F(T),
\end{array}
$$


where $T$ is the similarity variable, $F$ is the similarity function and $u$ is the group invariant solution of Eq.(1.1). Using Eqs.(4.7)-(4.8), Eq.(1.1) reduces to

$$
F_{T}=0
$$

On solving equation (4.9), we get $F=B_{2}$, which implies $u=B_{2}$, where $B_{2}$ is an arbitrary constant. In this case also, we obtained the trivial solution of Eq. (1.1.

Vector field (4) $V_{4}=V_{3}+V_{1}$

Corresponding to the infinitesimal generator $V_{4}=\frac{\partial}{\partial x}+t \frac{\partial}{\partial t}-u \frac{\partial}{\partial u}$, we have the following characteristic equation : $\frac{d x}{1}=\frac{d t}{t}=\frac{d u}{-u}$, thus by solving this characteristic equation, we obtained the group invariant solution as given below

$$
\begin{array}{r}
X=\frac{e^{x}}{t}, \\
u=\frac{F(X)}{t},
\end{array}
$$

with similarity variable $X$ and similarity function $F$. Now, by making use of Eqs. (4.11)- (4.13), Eq. (1.1) reduces to the following ODE:

(4.12)F $-X F_{X}+X F F_{X}+\mu\left[2 X F_{X}+4 X^{2} F_{X X}+X^{3} F_{X X X}\right]=0$.

Eq. (4.12) is a nonlinear ODE which can be solved numerically.

\section{Some More Exact Solutions of EWW Equation by Tanh Method}

\section{The Main Steps of Tanh Method :}

In this section, we discussed main steps of tanh method. The tanh method is first developed by Malfliet [21]. We will refer to Malfliet [21] 
and A. M. Wazwaz [22] for more details. This method is based on the fundamental concept that the traveling wave solution can be written in terms of tanh function. By using the tanh method one can find out exact traveling wave solutions or solitary solutions of nonlinear partial differential equations by converting them into nonlinear ordinary differential equations taking traveling wave transformation.

The main steps of the tanh method $[9,22,23]$ are as follows:

Step 1: Let us consider nonlinear partial differential equation (NPDE), given as

$$
H\left(u, u_{x}, u_{x x}, \ldots, u_{t}\right)=0
$$

where $\mathrm{H}$ is a polynomial in $u, u_{x}, u_{x x}, \ldots u_{t}$.

Step 2: We use the traveling wave transformation $u(x, t)=F(X), X=$ $k x+d t$, where $k$ and $d$ are the unknown parameters to be determined later. By making use of this transformation, the nonlinear PDE (2.1) gets converted into nonlinear ODE:

$$
I\left(F, F^{\prime}, F^{\prime \prime}, F^{\prime \prime \prime} \ldots\right)=0 .
$$

Step 3: Let us take the solution of Eq.(5.2) in the form finite series, given below:

$$
F(X)=\sum_{i=0}^{N} a_{i} Y^{i},
$$

where $N$ is a positive integer, which is to be determined by balancing the highest order nonlinear term with the highest order linear term in the resulting equation, and $Y=\tanh (X)$ is the new independent variable.

We replace all the derivatives in the resulting Eq.(5.2) with the following change of derivatives: 


$$
\begin{aligned}
\frac{d}{d X} & =\left(1-Y^{2}\right) \frac{d}{d Y}, \\
\frac{d^{2}}{d X^{2}} & =-2 Y\left(1-Y^{2}\right) \frac{d}{d Y}+\left(1-Y^{2}\right)^{2} \frac{d^{2}}{d Y^{2}}, \\
\frac{d^{3}}{d X^{3}} & =2\left(1-Y^{2}\right)\left(3 Y^{2}-1\right) \frac{d}{d Y}-6 Y\left(1-Y^{2}\right)^{2} \frac{d^{2}}{d Y^{2}}+\left(1-Y^{2}\right)^{3} \frac{d^{3}}{d Y^{3}} .
\end{aligned}
$$

Similarly, the other derivatives can be obtained.

In order to find the value of the index $N$, we use the following scheme:

$$
\begin{array}{ll}
F & \rightarrow N, \\
F^{n} & \rightarrow n N, \\
F^{\prime} & \rightarrow N+1, \\
F^{\prime \prime} & \rightarrow N+2, \\
F^{(s)} & \rightarrow N+s .
\end{array}
$$

Step 4: In this step, we substitute the value of $\mathrm{F}$ and all its derivatives into Eq.(5.2), then we collect the coefficients of $Y^{i} \quad(i=1,2,3, \ldots)$ and equating them to zero, we obtained system of nonlinear algebraic equations with unknown parameters $a_{i}, \quad i=1,2, \ldots, N$. This system of algebraic equations can be solved either manually or by any symbolic program such as MAPLE or MATHEMATICA. Thus, one can get the solitary wave solutions or traveling wave solution of Eq.(2.1) by putting the values of unknowns into Eq.(5.3).

\section{Implementation of Tanh Method on EWW Equation:}

By taking constants $c_{1}=0, c_{2}=-k, c_{3}=d$ in Eq. (3.4), we get infinitesimal generator: $V_{5}=d \frac{\partial}{\partial x}-k \frac{\partial}{\partial t}$, for which we have the following characteristic equation: $\frac{d x}{d}=\frac{d t}{-k}=\frac{d u}{0}$, thus by solving this characteristic equation, we obtained the following group invariant solution

$$
\begin{array}{r}
X=k x+d t, \\
u=F(X),
\end{array}
$$

with similarity variable $X=k x+d t$ which is in the form of traveling wave, where $\mathrm{k}$ and $\mathrm{d}$ are the unknown constants to be determined later. Now, by using the traveling wave transformation $X=k x+d t$ and Eqs.(5.5), Eq.(1.1) reduces to the following nonlinear ordinary differential equation:

$$
d F_{X}+k F F_{X}-\mu d k^{2} F_{X X X}=0 .
$$

On integrating Eq.(5.6) and taking integration constant zero, we obtain the following nonlinear ODE: 


$$
2 d F+k F^{2}-2 \mu d k^{2} F_{X X}=0 .
$$

Here, we apply tanh method for finding exact solution of Eq. (5.7)

On introducing a new variable $Y=\tanh (X)$, Eq.(5.7) is reduced to the equation:

$$
2 d F+k F^{2}-2 \mu d k^{2}\left[-2 Y\left(1-Y^{2}\right) F_{Y}+\left(1-Y^{2}\right)^{2} F_{Y Y}\right]=0
$$

Let us take the solution of Eq. (5.8) in the following form:

$$
F(Y)=\sum_{i=0}^{N} a_{i} Y^{i},
$$

where $a_{i} \quad(i=1,2, \ldots, N)$ and $N$ are the unknown parameters.

Now, we determine the parameter $\mathrm{N}$, by balancing the linear term of highest-order with the highest order nonlinear terms. Therefore, we balance $F^{2}$ and $F_{Y Y}$ and get: $2 N=N+2 \Rightarrow N=2$.

Therefore, the finite expression in Eq. (5.9) reduces to the following expression:

$$
F(Y)=a_{0}+a_{1} Y+a_{2} Y^{2}
$$

After putting the values of $F, F^{2}, F_{Y}$ and $F_{Y Y}$ in Eq.(5.8) and then equating the coefficients of $Y^{i}, \quad i=0,1,2,3,4$, we obtained the system of nonlinear algebraic equations as follows:

Coeff. of $Y^{0}: \quad 2 d a_{0}+k a_{0}^{2}-4 \mu d k^{2} a_{2}=0$,

Coeff. of $Y^{1}: \quad 2 d a_{1}+2 k a_{0} a_{1}+4 \mu d k^{2} a_{1}=0$,

Coeff. of $Y^{2}: \quad 2 d a_{2}+k\left(a_{1}^{2}+2 a_{0} a_{2}\right)+16 \mu d k^{2} a_{2}=0$,

Coeff. of $Y^{3}: \quad 2 k a_{1} a_{2}-4 \mu d k^{2} a_{1}=0$,

Coeff. of $Y^{4}$ : $k a_{2}^{2}-12 \mu d k^{2} a_{2}=0$.

By solving above system of nonlinear algebraic equations with the help of MAPLE 15, we obtain the following sets of unknown parameters:

Set 1. : $\mu=\mu, k=-\frac{1}{2}, a_{0}=6 d, a_{1}=0, a_{2}=-6 d$, 
Set 2. : $\mu=\mu, k=\frac{1}{2}, a_{0}=-6 d, a_{1}=0, a_{2}=6 d$,

Set 3. : $\mu=\mu, k=k, a_{0}=0, a_{1}=0, a_{2}=0$,

Set 4. : $\mu=\mu, k=k, a_{0}=-\frac{2 d}{k}, a_{1}=0, a_{2}=0$,

Set 5. : $\mu=\mu, k= \pm \frac{i}{2}, a_{0}=-2 d( \pm i), a_{1}=0, a_{2}=6 d( \pm i)$,

Therefore, the traveling wave solutions of Eq. (1.1) for $\mu=1$ are as follows:

\section{Corresponding to Set 1:}

$$
u(x, t)=6 d-6 d \tanh ^{2}\left(-\frac{x}{2}+d t\right)
$$

\section{Corresponding to Set 2:}

$$
u(x, t)=-6 d+6 d \tanh ^{2}\left(\frac{x}{2}+d t\right)
$$

\section{Corresponding to Set 3:}

$$
u(x, t)=0 .
$$

In this case, we found trivial solution of Eq. (1.1).

\section{Corresponding to Set 4:}

$$
u(x, t)=-\frac{2 d}{k},
$$

Clearly, it is a constant solution of Eq. (1.1).

\section{Corresponding to Set 5:}

$$
\begin{aligned}
& u(x, t)=-2 d i+6 d i \tanh ^{2}\left(\frac{i x}{2}+d t\right), \\
& u(x, t)=2 d i-6 d i \tanh ^{2}\left(-\frac{i x}{2}+d t\right) .
\end{aligned}
$$




\section{Power Series Solutions and Their Convergence}

In this section, we have obtained power series solution of Eq. (4.3). Let the Eq. (4.3) have the solution in the form of power series:

$$
F(X)=\sum_{n=0}^{\infty} C_{n} X^{n}
$$

Substituting Eq. (6.1) into Eq. (4.3), we get

$$
-\sum_{n=0}^{\infty} C_{n} X^{n}+\sum_{n=0}^{\infty} C_{n} X^{n} \sum_{n=1}^{\infty} C_{n} n X^{n-1}+\mu \sum_{n=2}^{\infty} n(n-1) C_{n} X^{n-2}=0
$$

The above equation can be rewritten as

$$
\begin{array}{r}
-C_{0}+C_{0} C_{1}+2 \mu C_{2}-\sum_{n=1}^{\infty} C_{n} X^{n}+\sum_{n=1}^{\infty} \sum_{k=0}^{n}(n+1-k) C_{k} C_{n+1-k} X^{n} \\
+\mu \sum_{n=1}^{\infty}(n+1)(n+2) C_{n+2} X^{n}=0 .
\end{array}
$$

On equating the coefficients of $x^{n}$ on both the sides, for $n=0$ in the above equation, we obtain

$$
C_{2}=\frac{C_{0}-C_{0} C_{1}}{2 \mu}
$$

and for $n \geq 1$, we obtain

$$
C_{n+2}=\frac{1}{\mu(n+1)(n+2)}\left[C_{n}-\sum_{k=0}^{n}(n+1-k) C_{k} C_{n+1-k}\right] .
$$

Therefore, the power series solution can be written as

$$
\begin{aligned}
F(X) & =C_{0}+C_{1} X+C_{2} X^{2}+\sum_{n=1}^{\infty} C_{n+2} X^{n+2} \\
& =C_{0}+C_{1} X+\frac{C_{0}-C_{0} C_{1}}{2 \mu} X^{2}+\sum_{n=1}^{\infty} \frac{1}{\mu(n+1)(n+2)} \\
& {\left[C_{n}-\sum_{k=0}^{n}(n+1-k) C_{k} C_{n+1-k}\right] X^{n+2} . }
\end{aligned}
$$

Therefore the power series solution of Eq. (1.1) is 


$$
\begin{aligned}
u(x, t) & =\frac{F(X)}{t} \\
& =\frac{1}{t}\left[C_{0}+C_{1} x+\frac{C_{0}-C_{0} C_{1}}{2 \mu} x^{2}+\sum_{n=1}^{\infty} \frac{1}{\mu(n+1)(n+2)}\right. \\
& {\left.\left[C_{n}-\sum_{k=0}^{n}(n+1-k) C_{k} C_{n+1-k}\right] x^{n+2}\right], }
\end{aligned}
$$

\section{Covergence of power series solution:}

Here, we proved the convergence of power series solution in Eq. (6.1) of Eq. (4.3). From Eq. (6.3), it is clear that $C_{0}$ and $C_{1}$ are two arbitrary constants, so let us take $C_{0}=\alpha \neq 0$ and $C_{1}=\beta$. Therefore, Eq.(6.3) implies $C_{2}=\frac{\alpha(1-\beta)}{2 \mu}$. Furthermore, from Eq. (6.4) we have $C_{3}=\frac{\beta-2 \alpha C_{2}-\beta^{2}}{6 \mu}$. In similar way, the other coefficients can be determined.

Now, from Eq.(6.4), we have

$$
\left|C_{n+2}\right| \leq M\left[\left|C_{n}\right|+\sum_{k=0}^{n}\left|C_{k}\right|\left|C_{n+1-k}\right|\right]
$$

where $M=\frac{1}{\mu}$ with $\mu$ being positive parameter. Now, we define new power series $\gamma=P(X)=\Sigma_{n=0}^{\infty} p_{n} X^{n}$ as

$p_{0}=\left|C_{0}\right|=|\alpha|, \quad p_{1}=\left|C_{1}\right|=|\beta|, \quad p_{2}=\left|C_{2}\right|$,

and

$p_{n+2}=M\left[p_{n}+\sum_{k=0}^{n} p_{k} p_{n+1-k}\right], \quad n=1,2, \cdots$.

It can be shown easily that $\quad\left|C_{n}\right| \leq p_{n}, \quad n=1,2,3, \cdots$, that is the series $\gamma=P(X)=\Sigma_{n=0}^{\infty} p_{n} X^{n}$ is the major series of the series in Eq. (6.1).

Now, we show that this major series has positive radius of convergence as follows:

$$
\begin{aligned}
P(X) & =p_{0}+p_{1} X+p_{2} X^{2}+\sum_{n=1}^{\infty} p_{n+2} X^{n+2} \\
& =p_{0}+p_{1} X+p_{2} X^{2}+M\left[\sum_{n=1}^{\infty} p_{n} X^{n+2}+\sum_{n=1}^{\infty} \sum_{k=0}^{n} p_{k} p_{n+1-k} X^{n+2}\right] \\
& =p_{0}+p_{1} X+p_{2} X^{2}+M\left[\left(\gamma-p_{0}+p_{0} p_{1}-p_{1} \gamma\right) X^{2}+\left(\gamma^{2}-2 \gamma p_{0}+p_{0}^{2}\right) X\right] .
\end{aligned}
$$

We have the implicit functional equation, written as

$$
\begin{aligned}
G(X, \gamma) & =\gamma-P(X) \\
& =\gamma-p_{0}-p_{1} X-p_{2} X^{2}-M\left[\left(\gamma-p_{0}+p_{0} p_{1}-p_{1} \gamma\right) X^{2}+\left(\gamma^{2}-2 \gamma p_{0}+p_{0}^{2}\right) X\right] .
\end{aligned}
$$

Clearly, $\mathrm{G}$ is analytic function in $(X, \gamma)$-plane and $G\left(0, p_{0}\right)=0, \quad G_{\gamma}^{\prime}\left(0, p_{0}\right)=$ $1 \neq 0$, therefore by the implicit function theorem [24], $\gamma=P(X)$ is analytic in the neighbourhood of the point $\left(0, p_{0}\right)$ and having positive radius of convergence. Hence, the power series is (6.1) convergent in the neighbourhood 
of the point $\left(0, p_{0}\right)$.

In this way, we obtained approximate power series solution of Eq. (4.3) as follows:

$$
\begin{aligned}
F(X) & =\alpha+\beta X+\frac{\alpha(1-\beta)}{2 \mu} X^{2} \\
& +\frac{\beta-2 \alpha C_{2}-\beta^{2}}{6 \mu} X^{3}+\frac{C_{2}-3 \alpha C_{3}-3 \beta C_{2}}{12 \mu} X^{4}+\cdots
\end{aligned}
$$

Therefore, an approximate power series solution of Eq.(1.1) is

$$
\begin{aligned}
u(x, t) & =\frac{F(X)}{t} \\
& =\frac{1}{t}\left[\alpha+\beta x+\frac{\alpha(1-\beta)}{2 \mu} x^{2}\right. \\
& \left.+\frac{\beta-2 \alpha C_{2}-\beta^{2}}{6 \mu} x^{3}+\frac{C_{2}-3 \alpha C_{3}-3 \beta C_{2}}{12 \mu} x^{4}+\cdots\right] .
\end{aligned}
$$

\section{Conclusion}

In this paper, we have applied the symmetry reduction technique on the equal width wave (EWW) equation by using the Lie Symmetry Transformation Method (STM). We performed STM on EWW equation and obtained infinitesimals of these equations. We found different infinitesimal generators by taking different values of constants $c_{i}, \quad i=1,2,3$, associated with the infinitesimal generators of EW wave equation. Based on the optimal system method, we considered all the group- invariant solutions to the reduced ODEs of Eq. (1). With the help of these infinitesimal generators, we get similarity variables and similarity functions. By utilizing these similarity variables and functions, we reduced nonlinear PDE (1) into nonlinear ODEs. Furthermore, the power series method have been applied on the reduced ODEs, and hence, we obtained exact and solitary wave solutions of Eq.(1). Also we have found exact explicit solutions of highly nonlinear EWW equation by using tanh method for the first time to the best of our knowledge. The obtained solutions are expressed in terms of the hyperbolic functions. We observed that, the STM is a powerful tool, it is more efficient and direct method to find the exact solutions of higher dimensional NPDEs.

\section{Acknowledgment}

The first author is thankful to the "University Grant Commission (UGC)", Government of India for the financial support under Sr. No. 2121541039 with Ref No. 20/12/2015(ii)EU-V. 


\section{References}

[1] M. Kaplan, A. Akbulut, and A. Bekir, "Exact travelling wave solutions of the nonlinear evolution equations by auxiliary equation method", Zeitschrift für naturforschung $A$, vol. 70, no. 11, pp. 969-974, Oct. 2015, doi: 10.1515/zna-2015-0122.

[2] P. J. Morrison, J. D. Meiss, and J. R. Cary, "Scattering of regularized-longwave solitary waves", Physica D: nonlinear phenomena, vol. 11, no. 3, pp. 324-336, Jun. 1984, doi: 10.1016/0167-2789(84)90014-9.

[3] J-H. He, "Variational iteration methoda kind of non-linear analytical technique: some examples", International journal of non-linear mechanics, vol. 34, no. 4, pp. 699-708, Jul. 1999, doi: 10.1016/S0020-7462(98)00048-1.

[4] E. Yusufoglu and A. Bekir, "Numerical solution of equal-width wave equation", Computers \& mathematics with applications, vol. 54, no. 7-8, pp. 1147-1153, Oct. 2007, doi: 10.1016/j.camwa.2006.12.080.

[5] R.-J. Cheng and H.-X. Ge, "Analysis of the equal width wave equation with the mesh-free reproducing kernel particle Ritz method", Chinese physics $B$, vol. 21, no. 10, Art. ID. 100209, Oct. 2012, doi: 10.1088/16741056/21/10/100209.

[6] A. Bihlo and R.O. Popovych, "Group classification of linear evolution equations", Journal of mathematical analysis and applications, vol. 448, no. 2, pp. 982-1005, Apr. 2017, doi: 10.1016/j.jmaa.2016.11.020.

[7] N. C. Roşca, A. V. Roșca, and I. Pop, "Lie group symmetry method for MHD double-diffusive convection from a permeable vertical stretching/shrinking sheet", Computers \& mathematics with applications, vol. 71, no. 8, pp. 16791693, Apr. 2016, doi: 10.1016/j.camwa.2016.03.006.

[8] Z. Y. Ma, H. L. Wu, and Q. Y. Zhu, "Lie symmetry, full symmetry group and exact solution to the (2+1)- dimemsional dissipative AKNS equation", Romanian journal of physics, vol. 62, no. 5-6, Art. ID. 114, 2017. [On line]. Available: https://bit.ly/3b1wCTS

[9] S. Sahoo and S. S. Ray, "Lie Symmetry analysis and exact solutions of (3+1) dimensional Yu-Toda-Sasa-Fukuyama equation in mathematical physics", Computers \& mathematics with applications, vol. 73, no. 2, pp. 253-260, Jan. 2017, doi: 10.1016/j.camwa.2016.11.016.

[10] S. Yang and C. Hua, "Lie symmetry reductions and exact solutions of a coupled KdV-Burgers equation", Applied mathematics and computation, vol. 234, pp. 579-583, May 2014, doi: 10.1016/j.amc.2014.01.044. 
[11] G. Wang, A. H. Kara, K. Fakhar, and J.V. Guzman, "Group analysis, exact solutions and conservation laws of a generalized fifth order KdV equation", Chaos, solitons \& fractals, vol. 86, pp. 8-15, May 2016, doi: 10.1016/j.chaos.2016.02.013.

[12] R. Arora and A. Chauhan, "Lie symmetry reductions and solitary wave solutions of modified equal width wave equation", International journal of applied and computational mathematics, vol. 4, no. 5, Art. ID. 122, Oct. 2018, doi: 10.1007/s40819-018-0557-z.

[13] A. F. Cheviakov and J. F. Ganghoffer, "Symmetry properties of two dimensional Ciarlet-Mooney-Rivlin constitutive models in nonlinear elastodynamics", Journal of mathematical analysis and applications, vol. 396, no. 2, pp. 625-639, Dec. 2012, doi: 10.1016/j.jmaa.2012.07.006.

[14] Y. Yildirim, E. Yaşar, H. Triki, Q. Zhou, S.P. Moshokoa, M.Z. Ullah, A. Biswas, and M. Belić, "Lie symmetry analysis and exact solutions to N-coupled non-linear Schrödinger's equations with kerr and parabolic law nonlinearities", Romanian journal of physics, vol. 63, no. 1-2, Art. ID. 103, 2018. [On line]. Available: https://bit.ly/2RL01tO

[15] H. Liu and J. Li, "Lie symmetry analysis and exact solutions for the short pulse equation", Nonlinear analysis: theory, methods \& applications, vol. 71, no. 5-6. pp. 2126-2133, Sep. 2009, doi: 10.1016/j.na.2009.01.075.

[16] P. J. Olver, Applications of lie groups to differential equations. New York, NY: Springer-Verlag, 1993.

[17] G. Bluman, A. Cheviakov, and S. Anco, Applications of symmetry methods to partial differential equations. New York, NY, 2010, doi: 10.1007/978-0387-68028-6.

[18] G. W. Bluman and J.D. Cole, Similarity methods for differential equations. New York, NY: Springe, 1974.

[19] L. V. Ovsiannikov, Group analysis of differential equations. New York, NY: Academic Press, 1982.

[20] N. Goyal, A. M. Wazwaz and R. K. Gupta, "Applications of maple software to derive exact solutions of generalized fifth-order Korteweg-De Vries equation with time-dependent coefficients", Romanian reports in physics, vol. 68, no. 1, pp. 99-111, 2016. [On line]. Available: https://bit.ly/3aZaIR8

[21] W. Malfliet, "Solitary wave solutions of nonlinear wave equations", American journal of physics, vol. 60, no. 7, pp. 650-654, Jul. 1992, doi: 10.1119/1.17120.

[22] A. M. Wazwaz, Partial differential equation and solitary wave theory, Berlin: Springer, 2009, doi: 10.1007/978-3-642-00251-9. 
[23] K. Raslan and Z. F. A. Shaeer, "The Tanh methods for the Hirota equations", International journal of computer applications, vol. 107, no. 11, pp. 5-9, Dec. 2014, doi: 10.5120/18793-0134.

[24] W. Rudin, Principles of mathematical analysis, 3rd ed., Beijing: China Machine Press, 2004. 